\title{
SOME INEQUALITIES ON THE PERRON EIGENVALUE AND EIGENVECTORS FOR POSITIVE TENSORS
}

\author{
QingBing LiU, CHAOQIAN Li AND CHENGYi ZHANG
}

Abstract. In this paper, we obtain new bounds for the maximal eigenvalue and eigenvectors of positive tensors and compare these bounds with the known bounds. Numerical experiments are given to validate the efficiency of our new bounds.

Mathematics subject classification (2010): 15A18, 15A69, 65A69, 65F10.

Keywords and phrases: Positive tensors, Perron-Frobenius theory, maximal eigenvalue bounds, maximal eigenvectors bounds.

\section{REFERENCES}

[1] L. QI, Eigenvalues of a real supersymmetric tensor, J. Symbolic Comput, 40, 6 (2005), 1302-1324.

[2] L. H. LIM, Singular values and eigenvalues of tensors, a variational approach, in: proceedings 1st IEEE international Workshop on computational Advances of Multitensor Adaptive Processing, 2005, $129-132$.

[3] L. QI, G. YU AND E. WU, Higher order positive semidefinite diffusion tensor imaging, SIAM J. Imaging Sci, 3, 3 (2010), 1-32.

[4] L. H. Lim, Multilinear Pagerank: Measuring Higher Order Connectivity in Linked Objects, The internet: Today and Tomorrow, 2005.

[5] J. Cooper AND A. Dutle, Spectra of uniform hypergraphs, Linear Algebra Appl, 436, 9 (2012), 3268-3292.

[6] K. C. ChANG AND T. ZhANG, On the uniqueness and nonuniqueness of the Z-eigenvector for transition probability tensors, J. Math Anal Appl, 408, 2 (2013), 1969-1972.

[7] A. LI, L. QI AND B. ZHANG, E-characteristic polynomials of tensors, Communications Math Sci, 11, (2013), 123-143.

[8] S. HU AND L. QI, Algebraic connectivity of an even uniform hypergraph, J. Combinatorial Optimization, 24, 4 (2012), 1-137.

[9] K. C. Chang, K. Pearson And T. Zhang, Perron-Frobenius theorem for nonnegative tensors, Communications Math Sci, 6, (2008), 253-279.

[10] W. Li AND M. NG, On the limiting probability distribution of a transition probability tensor, Linear Multilinear Algebra, DOI: 10.1080/03081087.2013.777436.

[11] H. Minc, Nonnegative Matrices, New York: John Wiley and Sons, Inc, 1988.

[12] Y. YANG AND Q. YANG, Further results for Perron-Frobenius theorem for nonnegative tensors, SIAM J. Matrix Anal Appl, 31, 5 (2010), 373-378.

[13] A. M. Ostrows KI, On the eigenvector belonging to the maximal root of a nonnegative matrix, Proc. Edinburgh Math. Soc, 12, (1960/1961), 107-112.

[14] H. SCHNEIDER, Note on the fundamental theorem on irreducible nonnegative matrices, Proc. Edinburgh Math. Soc, 11, (1958), 127-130.

[15] Z. WANG AND W. WU Bounds for the greatest eigenvalue of positive tensors, J. Industrial Management Optimization, 10, 4 (2014), 1031-1039.

[16] K. C. Chang, K. Pearson And T. Zhang, Some variational principles for Z-eigenvalues of nonnegative tensors, Linear Algebra Appl, 438, 11 (2013), 4166-4182.

[17] Y. LiU, G. ZHOU AND N. F. IBRAHIM, An always convergent algorithm for the largest eigenvalue of an irreducible nonnegative tensor, J. Comput. Appl Math, 235, 1 (2010), 286-292. 
[18] M. NG, L. QI AND G. ZHOU, Finding the largest eigenvalue of a nonnegative tensor, SIAM J. Matrix Anal Appl, 31, 3 (2009), 1090-1099.

[19] L. ZHANG AND L. QI, Linear convergence of an algorithm for computing the largest eigenvalue of a nonnegative tensor, Numer Linear Algebra Appl, 19, (2012), 830-841.

[20] S. Friedland, S. Gaubert And H. Li, Perron-Frobenius theorem for nonnegative multilinear forms and extensions, Linear Algebra Appl, 438, 2 (2013), 738-749.

[21] K. C. Chang, K. PeArson And T. ZHAng Primitivity, the convergence of the NQZ method, and the largest eigenvalue for nonnegative tensors, SIAM J. Matrix Anal Appl, 32, 3 (2011), 806-819.

[22] L. ZHANG, L. QI AND Y. XU, Linear convergence of the LZI algorithm for weakly positive tensors, J. Comput Math, 30, 1 (2013), 24-33.

[23] G. ZHOU, L. QI AND S. WU, Efficient algotithms for computing the largest eigenvalue of a nonnegative tensor, Frontiers Math China, 8, (2013), 155-168. 